\title{
Long-Term Effect of Early Phacoemulsification in Primary Angle Closure Glaucoma Patients with Cataract: A I0-Year Follow-Up Study
}

\author{
Hazem Helmy (10 \\ Glaucoma and Optic Nerve Disease \\ Department, Research Institute of \\ Ophthalmology, Giza, Egypt
}

Correspondence: Hazem Helmy Glaucoma and Optic Nerve Disease Department, Research Institute of Ophthalmology, 2 El Ahram Street, Giza, 12577, Egypt

Tel +20122400499I; +20223I5228

Email hazem.helmy@yahoo.com
Purpose: To assess the 10-year effects of early phacoemulsification with intraocular lens (IOL) implantation in primary angle closure glaucoma (PACG) patients with cataract.

Patients and Methods: This prospective cohort study included 102 eyes of 102 patients with PACG. All patients had coexisting cataracts compromising vision. Patients underwent phacoemulsification and foldable IOL implantation. The main outcome measures were anterior chamber depth (ACD), angle width, value of intraocular pressure (IOP), and number of medications needed postoperatively and during follow-up.

Results: Half (53\%) of the patients were female, with ages ranging from 55 to 73 with a mean of $59.82 \pm 5.19$ years. Mean IOP decreased significantly from $22.15 \pm 2.08 \mathrm{mmHg}$ at baseline to $14.08 \pm 2.13 \mathrm{mmHg}$ postoperatively $(\mathrm{p}<0.05$ ). The ACD increased from $2.2 \pm 0.21$ preoperatively to $3.73 \pm 0.25$ postoperatively $(p<0.001)$. Nasal angle width increased postoperatively to $40.05 \pm 2.09$ compared to the preoperative value of $16.02 \pm 2.08(\mathrm{p}<0.001)$. Temporal angle width increased from $13.05 \pm 2.07$ to $41.9600 \pm 1.94(p<0.001)$. Antiglaucoma treatment significantly decreased postoperatively $(\mathrm{p}<0.001)$. A significant positive correlation was detected between ACD and angle width, while a negative correlation was detected between IOP and both ACD and angle width $(\mathrm{p}<0.001)$. There was also a significant negative correlation between postoperative angle width and IOP $(\mathrm{p}<0.001)$. Preoperative lens thickness was positively correlated with preoperative IOP and number of medications, while it was negatively correlated with preoperative AC depth and angle width. Preoperative lens thickness positively correlated with postoperative IOP and medications. Complete and qualified success was achieved in $69.65 \%$ and $30.4 \%$ of cases, respectively, while $2.9 \%$ failed to be controlled. Visual acuity significantly improved from $0.17 \pm 0.1$ to $0.9 \pm 0.08$ $(\mathrm{p}<0.001)$. All parameters showed high stability throughout the follow-up period.

Conclusion: Phacoemulsification with IOL implantation is a safe and effective early modality for long-term control of IOP in PACG patients with coexisting cataract. The effects can persist for at least 10 years.

Keywords: primary angle closure glaucoma, early phacoemulsification, anterior chamber depth, angle width, UBM

\section{Introduction}

Primary angle closure glaucoma (PACG) disease is exceedingly common in African-American patients and is responsible for about $50 \%$ of glaucoma blindness. ${ }^{1}$ It usually affects older patients and coincides with cataract appearance. $^{2}$ PACG has an unclear pathogenetic mechanism and multi-factorial pathophysiology. ${ }^{3}$ The lens is a continuously growing organ that may be the 
cause of pupillary block mechanism in most cases. $^{4-7}$ Plateau iris and iris abnormalities constitute non- pupillary block mechanisms that may be aggravated, and increased lens size may also play a role. In plateau iris, the anterior chamber depth (ACD) is normal when the iris is plane. On ultrasound bio microscopy (UBM), this condition appears as a flat and anteriorly situated iris surface with ciliary processes. The iris root is steep, short, and thick. An absence of ciliary sulcus in addition to anterior angulation of peripheral iris are present. Plateau iris can be diagnosed by gonioscopy as a double hump sign and deep AC center despite shallow periphery. Plateau iris syndrome is a form of PACG that results from a large or anteriorly positioned ciliary body that leads to mechanical obstruction of the trabecular meshwork. This condition can be recognized by lack of angle opening as a response to laser peripheral iridotomy (LPI) with intraocular pressure (IOP) elevation after dilatation. ${ }^{8-10}$ Primary angle closure suspect (PACS) is defined when $\geq 180$ degrees of iridotrabecular contact is present without peripheral anterior synechia (PAS), intraocular pressure (IOP) elevation, or optic nerve damage. This definition changes to primary angle closure (PAC) when IOP becomes elevated in PACS. PACG becomes evident when IOP and glaucomatous optic disc changes appear in PAC cases. ${ }^{11,12}$ Neodymium-YAG LPI is considered the primary line of treatment for such cases, but it is not successful in some cases. ${ }^{13,14}$ Failure can be due to anatomical changes in PACG eyes, such as thick iris or swollen and anteriorly positioned lens that pushes the iris towards the trabecular meshwork and induces iris crowding. ${ }^{15}$ Laser iridoplasty may be an adjunctive approach in some cases, but trabeculectomy is usually reserved for cases with extensive PAS that do not respond well to these simple methods of treatment. ${ }^{14,16}$ Shallow anterior chamber (AC), hypotony, choroidal detachment, and malignant glaucoma are common complications of trabeculectomy in these cases. ${ }^{17,18} \mathrm{~A}$ crowded anterior segment is the most obvious predisposing factor. ${ }^{19}$ Quigelly et al concluded that the AC angle does not return to the physiological state after LPI, with the exception of iris flattening. This may have been due to forward movement of the iris lens diaphragm with secondary iridotrabecular appositional contact that ends with synechia and angle closure. ${ }^{13,19}$ An attempt to deepen the AC, opening the angle, and preserving trabecular meshwork function with phacoemulsification at an early stage may be a good choice. $^{20-22}$ This could help avoid trabeculectomy or phacotrabeculectomy complications that may be highly aggressive in this type of patient. ${ }^{23-26}$ Early detection and treatment can prevent progression and decrease morbidity of the trabeculum and maintain the angles of the AC and optic nerve. ${ }^{19}$ Two groups concluded that phacoemulsification might be an appropriate primary modality for PACG management. ${ }^{20,28}$ Early intervention by phacoemulsification when the PAS is $\leq 180$ may be the ideal stage for intervention for permanent preservation of filtering trabeculum, but long-term follow-up results are lacking. ${ }^{29} \mathrm{We}$ performed a 10-year follow-up study of outcomes in patients with PACG (controlled or uncontrolled with medical treatment, PAS $\leq 180$ degrees of the AC angle, and reasonable cataract) who underwent early intervention with phacoemulsification.

\section{Materials and Methods}

A prospective cohort long term follow-up study that included 102 eyes of 102 patients with PACG on medical treatment and reasonable cataract. Anterior chamber angle grade 2 or more according to Shaffer grading scale in 3 or all quadrants is an essential parameter in cases to be eligible for the study. All patients underwent phacoemulsification plus foldable IOL implantation.

\section{Preoperative Evaluation}

All patients underwent full ophthalmological examination that included best corrected visual acuity (BCVA) by Landolt $\mathrm{C}$ optotype chart converted to $\log$ MAR scale, IOP measurement with Goldman applanation tonometry (Haag-Streit AT 900, Swiss made), two values were assessed and the mean value was used for statistical analysis, gonioscopy with Zeiss 4 mirror gonio-lens (Model OPDSG, Ocular instruments, USA) with documentation of grading in all 4 quadrants according to Shaffer grading scale, optic disc examination with slit lamp bio microscopy. Fundoscopy was performed with 90 diopter lens and slit-lamp bio microscopy when possible; otherwise, B scan ultrasonography was performed. Angle examination with ultrasound bio microscopy (Sonomed UBM $35 \mathrm{MHZ}$ transducer) was performed that included angle width, anterior and posterior chamber evaluation in addition to iris lens relation. A scan ultrasonography (Sonomed, USA) was used to assess AC depth and lens thickness.

\section{Postoperative Follow}

All patients were followed in the first postoperative day, week and then at 1,3.6,12 months and then every 6 months 
for 5 years then yearly for 5 years. Ultrasound biomicroscopy was performed three months postoperatively to assess angle width. A scan ultrasonography was used to assess postoperative AC depth. Number of anti-glaucoma treatments were recorded. Success was defined as IOP to become less than $21 \mathrm{mmHg}$ and more than $6 \mathrm{mmHg}$ which can be documented as complete when this task was achieved without medical treatment. Success was defined as qualified when IOP became less than $21 \mathrm{mmHg}$ with aid of medical treatment while failure was prescribed when IOP became more than $21 \mathrm{mmHg}$ even with medical treatment. The study was performed at Research Institute of Ophthalmology (RIO) Egypt in accordance with the tenets of the declaration of Helsinki 1975 (The 1983 revision). The research Committee of RIO approved and revised this study. An informed consent was signed by all participants of this study after a thorough explanation of procedure.

\section{Inclusion Criteria}

PACG patients with PAS of 180 degrees or less, BCVA less than 0.5 due to cataract. Type of cataract was nuclear grade 1 and 2 in addition to anterior cortical and posterior sub-capsular cataract.

\section{Exclusion Criteria}

Patients with history of intraocular surgery, patients with ocular diseases that may be associated with altered normal anatomy of anterior segment as iris, ciliary body mass, cyst or trauma. Patients with any type of secondary angle closure glaucoma. Patients with congenital, traumatic, neovascular and uveitis glaucoma were excluded from this study.

\section{Surgical Technique}

Oral acetazolamide and intravenous mannitol infusion were given preoperatively in order to dehydrate the vitreous and regress forward movement of iris lens diaphragm preoperatively. All surgical procedures were done by the same surgeon $(\mathrm{HH})$. Local peribulbar anesthesia was the mode of anesthesia in all cases using 4-6 mL of 1:1 mixture of (lidocaine hydrochloride $2 \%$ and bupivacaine $0.5 \%$ ) in addition to $50 \mathrm{IU}$ hyaluronidase. Intravenous sedation was administered whenever needed. A three-step clear corneal incision was performed followed by two angled side ports. AC was inflated with viscoelastic (Healon GV). Cystotome forceps was used to create a continuous curvilinear capsulorhexis then hydro dissection and hydro-delineation were
Table I Baseline Data of the Studied Patients $(n=102)$

\begin{tabular}{|l|l|l|}
\hline & $\mathbf{N}=102$ & $\%$ \\
\hline Gender: & 53 & \\
Female & 49 & 52 \\
Male & & 48 \\
\hline Gonioscopy: & 12 & \\
Angle grade 2 in 3 quadrants & 90 & 11.8 \\
Angle grade 2 in all quadrants & 98.2 \\
\hline Glaucoma mechanism: & 62 & \\
Pupillary block & 40 & 61 \\
Plateau iris & & 39 \\
\hline Age (years): & $59.82 \pm 5.186$ & \\
Mean \pm SD & $55-73$ & \\
Min - Max & & \\
\hline Lens thickness: & $3.81 \pm 0.21$ & \\
Mean \pm SD & $3.4-4.2$ & \\
Min - Max & & \\
\hline C/D ratio: & $0.4 \pm 0.15$ & \\
Mean \pm SD & $0.1-0.7$ & \\
Min - Max & & \\
\hline
\end{tabular}

performed. In the bag phacoemulsification using divide and conquer technique was done then cortical aspiration was achieved. An acrylic intraocular lens (AcrySof, Alcon Laboratories Inc.) was implanted. All surgically complicated cases were excluded from the study. All cases received postoperative medications that included Gatifloxacin $0.3 \% 6$ times daily for 3 weeks and prednisolone acetate 1\% 6 times daily for 1 week followed by gradual tapering along 1 month. Anti-glaucoma treatment was given when needed according to IOP measurement.

\section{Data Analysis}

Data analysis was performed using the software SPSS (Statistical Package for the Social Sciences) version 20. Quantitative variables were described using their means

Table 2 Change in Visual Acuity in the Studied Patients Pre and Postoperatively

\begin{tabular}{|l|l|l|l|l|}
\hline \multirow{2}{*}{ Time } & \multicolumn{2}{|l|}{ AC Depth } & \multicolumn{2}{l|}{ Test } \\
\cline { 2 - 5 } & $\begin{array}{l}\text { Mean } \pm \\
\text { SD }\end{array}$ & $\begin{array}{l}\text { Median } \\
\text { (Range) }\end{array}$ & Wx & p \\
\hline $\begin{array}{l}\text { Preoperatively } \\
\text { I month } \\
\text { postop }\end{array}$ & $0.17 \pm 0.1$ & $0.1(0.05-0.4)$ & & \\
$0.9 \pm 0.08$ & $0.9(0.7-1.0)$ & -8.97 & $<0.00$ I** \\
\hline
\end{tabular}

Note: ${ }^{* *} p \leq 0.00$ I is statistically highly significant.

Abbreviation: $\mathrm{Wx}$, Wilcoxon signed rank test. 


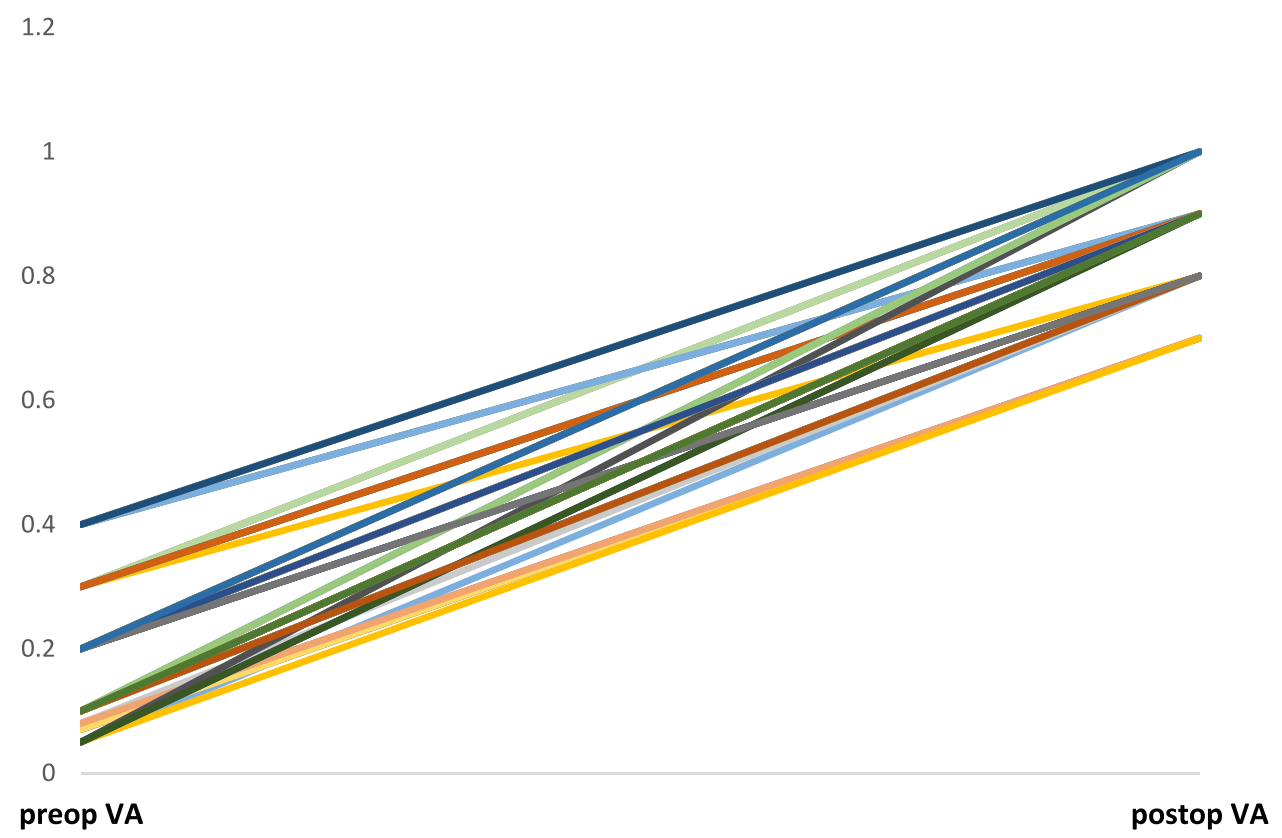

Figure I Multiple line graph showing significant increase in visual acuity postoperatively as compared to preoperative values among the studied patients.

and standard deviations. Categorical variables were described using their absolute frequencies to compare the proportion of categorical data. Kolmogorov-Smirnov (distribution-type) and Levene (homogeneity of variances) tests were used to verify assumptions for use in parametric tests. To measure the change in continuous variable over two points of time within the same group, paired sample $t$ test was used. To measure change in continuous parametric data over more than two points of time in the same group, repeated measure ANOVA was used for normally distributed data. Wilcoxon signed rank test was used to measure change in continuous non-parametric variable within the same group over two points of time. Marginal homogeneity test was used to measure change in paired ordinal data. Pearson

Table 3 Change in Medications in the Studied Patients Pre and Postoperatively

\begin{tabular}{|l|l|l|l|l|}
\hline \multirow{4}{*}{ Medication } & \multicolumn{2}{|l|}{ Time } & \multicolumn{2}{l|}{ Test } \\
\cline { 2 - 5 } & Preop & Postop & MH & P \\
\cline { 2 - 3 } & N=102 (\%) & N=102 (\%) & & \\
\hline No & $0(0)$ & $71(69.6)$ & & \\
I drug & $0(0)$ & $11(10.8)$ & 9.507 & $0.00 I^{* *}$ \\
2 drugs & $37(36.3)$ & $19(18.6)$ & & \\
3 drugs & $65(63.7)$ & I (1) & & \\
\hline
\end{tabular}

Note: $* * x \leq 0.001$ is statistically highly significant. Abbreviation: $\mathrm{MH}$, marginal homogeneity test. correlation coefficient was used to measure strength and direction of correlation between continuous parametric variables. The level statistical significance was set at $\mathrm{P}<0.05$. Highly significant difference was present if $\mathrm{p} \leq 0.001$.

\section{Results}

Similar numbers of males and females were included ( $48 \%$ and $53 \%$, respectively). Their ages ranged from 55 to 73 years with a mean of 59.82 years. Lens thickness ranged from 3.4 to $4.2 \mathrm{~mm}$ with a mean of $3.81 \mathrm{~mm}$. The C/D ratio ranged from 0.1 to 0.7 with a mean of 0.4 . Plateau iris was detected in 40 (39\%) of cases, while pupillary block was the mechanism of angle closure in $62(61 \%)$ of cases. Grade I angle width in all quadrants was present in $90(88.2 \%)$ of cases, while grade I or higher was detected in only two quadrants in 12 $(11.8 \%)$ of cases (Table 1).

There was statistically significant improvement in postoperative visual acuity as compared to preoperative values (Table 2, Figure 1). We observed a statistically significant decrease in the number of medications used after surgery (Table 3, Figure 2). There was also a statistically significant decrease in IOP 1 month postoperatively compared to baseline. This IOP level persisted until 30 months postoperatively; then, it non-significantly decreased and increased again at 36 months to reach a stable level throughout the 10year follow-up period (Table 4, Figure 3). 


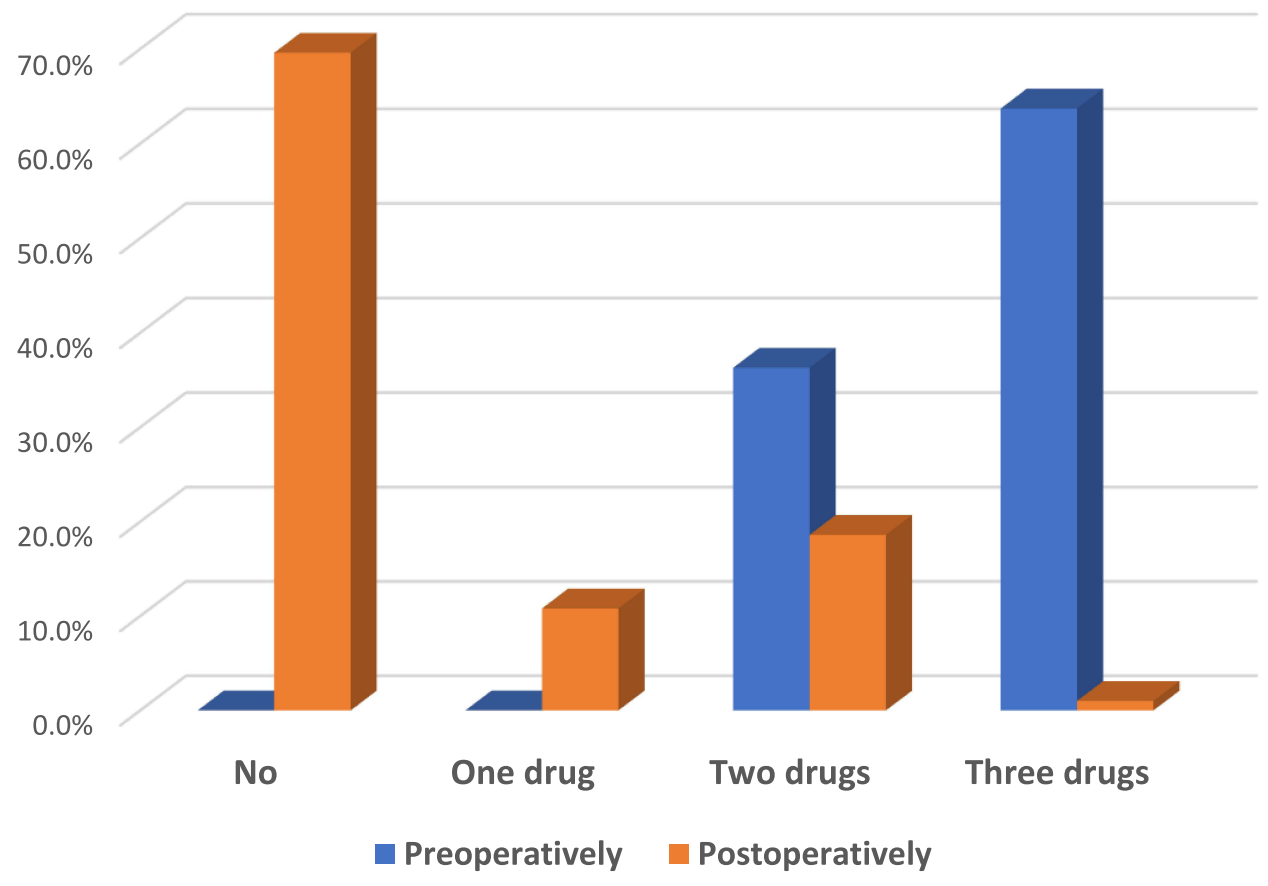

Figure 2 Multiple bar chart showing change in medications postoperatively compared to preoperative value among the studied patients.

There was a statistically significant increase in ACD 1 month postoperatively compared to baseline. No further improvement was noted at 3 months postoperatively, and ACD remained stable thereafter (Table 5, Figure 4). We observed statistically significant increases in nasal and temporal angle width after surgery (Table 6).

ACD was significantly positively correlated with both nasal and temporal angle widths, which were also positively correlated with each other. Conversely, there were statistically significant negative correlations between IOP and ACD and nasal and temporal angle widths. IOP and number of medications also showed a significant positive correlation. There was a statistically significant negative correlation between number of medications and ACD and both nasal and temporal angle widths (Table 7, Figure 5). We observed non-significant correlations between ACD and nasal and temporal angle widths or IOP (Table 8, Figure 6).

There was a significant positive correlation between the nasal and temporal angle widths. There were statistically negative correlations between nasal angle width and IOP (Table 9) and between temporal angle and IOP. We found a significant positive correlation between IOP and number of medications, and significant negative correlations between number of medications and both the nasal and temporal angle widths. There was also a statistically significant negative correlation between lens thickness and preoperative ACD and nasal and temporal angle widths. There was a significant positive correlation between lens thickness and both preoperative IOP and number of medications (Table 8).

We found a significant negative correlation between lens thickness and the postoperative nasal and temporal angles. There were statistically significant positive correlations between lens thickness and both postoperative IOP and the number of medications used. The positive correlation between lens thickness and postoperative ACD was not significant (Table 9).

Complete success was observed in $69.6 \%$ of cases at the end of the study. Three patients required glaucoma surgery during the 10-year follow-up period (Table 10, Figure 7). Complications associated with phacoemulsification included corneal edema, uveitis, hyphema, macular edema, malignant glaucoma, and IOP spike. All resolved completely with conservative medical treatment within 2 to 3 weeks, and no further surgical intervention was required (Table 11).

\section{Discussion}

PACG is a group of disorders caused by different mechanisms. Mechanical blockage of the trabecular meshwork by peripheral iris is the most common sign of this disease. Relative pupillary block in most cases and plateau iris in a minority are the most prominent mechanisms. ${ }^{4,7,11}$ 
Table 4 Change in IOP in the Studied Patients Pre and Postoperatively

\begin{tabular}{|l|l|l|}
\hline \multirow{2}{*}{ Time } & \multicolumn{2}{|l|}{ IOP } \\
\cline { 2 - 3 } & Mean \pm SD & Range \\
\hline Preoperatively & $22.15 \pm 2.08$ & $18-27$ \\
I Month postop & $14.08 \pm 2.13$ & $10-19$ \\
\hline P (t) & $<0.001 * *$ & \\
\hline 3 Months postop & $14.08 \pm 2.13$ & $10-19$ \\
6 Months postop & $14.08 \pm 2.13$ & $10-19$ \\
9 Months postop & $14.08 \pm 2.13$ & $10-19$ \\
12 Months postop & $14.08 \pm 2.13$ & $10-19$ \\
18 Months postop & $14.08 \pm 2.13$ & $10-19$ \\
24 Months postop & $14.08 \pm 2.13$ & $10-19$ \\
30 Months postop & $13.99 \pm 2.11$ & $10-19$ \\
36 Months postop & $14.08 \pm 2.13$ & $10-19$ \\
42 Months postop & $14.08 \pm 2.13$ & $10-19$ \\
48 Months postop & $14.08 \pm 2.13$ & $10-19$ \\
60 Months postop & $14.08 \pm 2.13$ & $10-19$ \\
6 Years postop & $14.08 \pm 2.13$ & $10-19$ \\
7 Years postop & $14.08 \pm 2.13$ & $10-19$ \\
8 Years postop & $14.08 \pm 2.13$ & $10-19$ \\
9 Years postop & $14.08 \pm 2.13$ & $10-19$ \\
10 Years postop & $14.08 \pm 2.13$ & $10-19$ \\
\hline P (f) & 0.32 & \\
\hline
\end{tabular}

Note: $F$ repeated measure ANOVA. ${ }^{*} \mathrm{p} \leq 0.00 \mathrm{I}$ is statistically highly significant. Abbreviation: $t$, paired sample $t$ test.

Early detection and intervention to stop these events can be highly beneficial in controlling IOP and preserving visual function. ${ }^{4,8,29}$ Gonioscopy is the gold standard for diagnosing angle closure. A 360 degree assessment can provide information about PAS in addition to differentiation between oppositional and synechial angle closure when indentation was performed. UBM is a useful tool to assess relationships between various anterior segment characteristics such as $\mathrm{AC}$ angle, iris, posterior chamber zonules, ciliary body, and crystalline lens. ${ }^{6,16,31,32}$ It is an extremely efficient tool to assess angle closure glaucoma dynamics in patients with plateau iris syndrome and pupillary block glaucoma. ${ }^{10}$

LPI is the cornerstone in primary approach of these cases. This treatment helps equalize pressure between the anterior and posterior chambers, flattens the iris, and widens the $\mathrm{AC}$ angle, particularly in patients with $\leq 180$ degrees PAS. ${ }^{18,19,30,31,33}$ This procedure may not be completely effective due to another continuously changing parameter in the form of crystalline lens that undergoes aging processes. Increased lens size and anteroposterior diameter can aggravate anterior segment crowding and cause further AC shallowing. ${ }^{6}$ The net effect is IOP elevation that has a negative impact on optic nerve health. Consequently, a lens-induced mechanism in the development of PACG gained a wide acceptance in recent years. ${ }^{4,6,8,19,29,34}$ Indeed, we observed a negative correlation between lens thickness and both $\mathrm{AC}$ angle width and depth. A negative correlation was also detected between angle width and both IOP and the number of anti- glaucoma drugs. This is supported by the findings of George
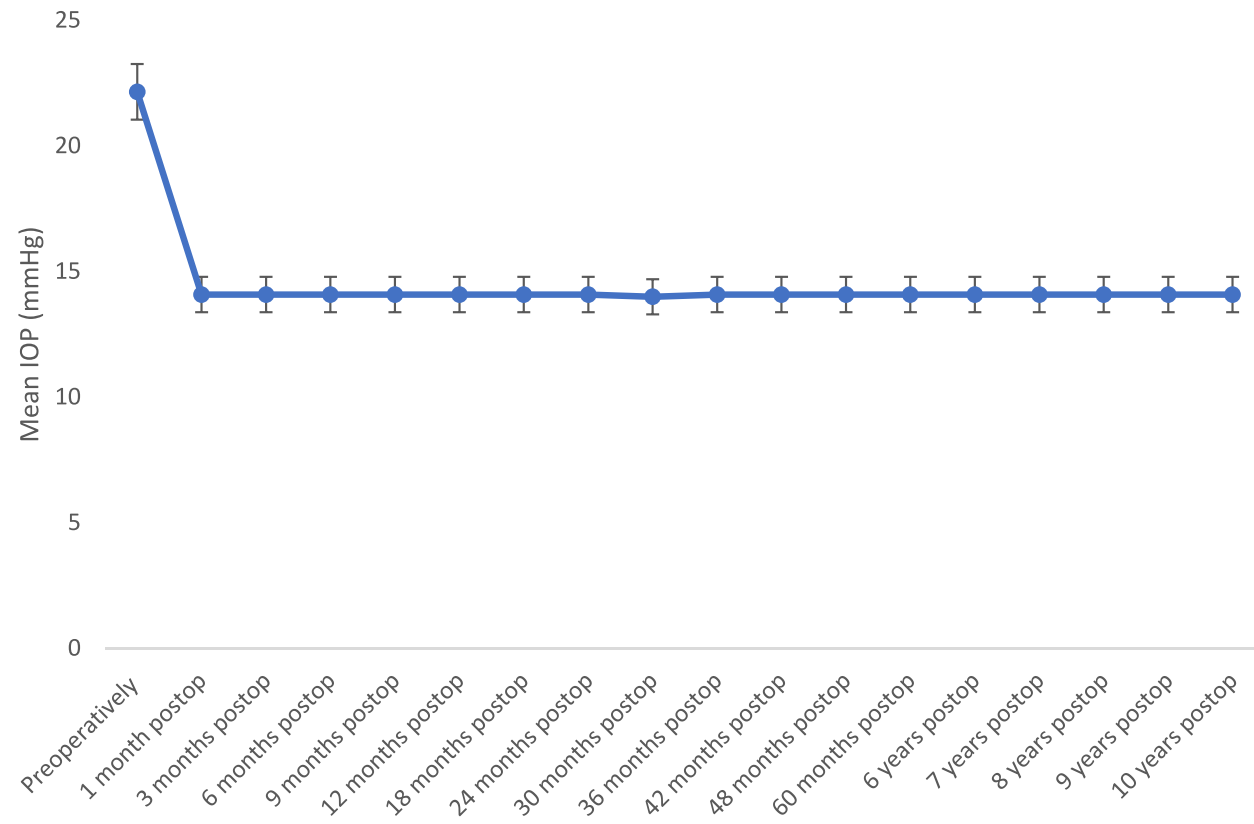

Figure 3 Line graph showing change in IOP pre and postoperatively among the studied patients. 
Table 5 Change in AC in the Studied Patients Pre and Postoperatively

\begin{tabular}{|c|c|c|}
\hline \multirow[t]{2}{*}{ Time } & \multicolumn{2}{|l|}{ AC Depth } \\
\hline & Mean \pm SD & Range \\
\hline Preoperatively & $2.2 \pm 0.21$ & $1.8-2.6$ \\
\hline I Month postop & $3.7 \pm 0.25$ & $2.1-3.79$ \\
\hline$P(t)$ & \multicolumn{2}{|l|}{$<0.001 * *$} \\
\hline 3 Months postop & $3.72 \pm 0.16$ & $2.1-3.79$ \\
\hline 6 Months postop & $3.72 \pm 0.16$ & $2.1-3.79$ \\
\hline 9 Months postop & $3.72 \pm 0.16$ & $2.1-3.79$ \\
\hline 12 Months postop & $3.72 \pm 0.16$ & $2.1-3.79$ \\
\hline I8 Months postop & $3.72 \pm 0.16$ & $2.1-3.79$ \\
\hline 24 Months postop & $3.72 \pm 0.16$ & $2.1-3.79$ \\
\hline 30 Months postop & $3.72 \pm 0.16$ & $2.1-3.79$ \\
\hline 36 Months postop & $3.72 \pm 0.16$ & $2.1-3.79$ \\
\hline 42 Months postop & $3.72 \pm 0.16$ & $2.1-3.79$ \\
\hline 48 Months postop & $3.72 \pm 0.16$ & $2.1-3.79$ \\
\hline 60 Months postop & $3.72 \pm 0.16$ & $2.1-3.79$ \\
\hline 6 Years postop & $3.72 \pm 0.16$ & $2.1-3.79$ \\
\hline 7 Years postop & $3.72 \pm 0.16$ & $2.1-3.79$ \\
\hline 8 Years postop & $3.72 \pm 0.16$ & $2.1-3.79$ \\
\hline 9 Years postop & $3.72 \pm 0.16$ & $2.1-3.79$ \\
\hline 10 Years postop & $3.72 \pm 0.16$ & $2.1-3.79$ \\
\hline$P(F)$ & \multicolumn{2}{|l|}{0.17} \\
\hline
\end{tabular}

Note: $\mathrm{F}$ repeated measure ANOVA. **p $\leq 0.001$ is statistically highly significant. Abbreviation: $t$, paired sample $t$ test.

et al who reported shorter axial length, shallower AC, and greater lens thickness in PACG compared to normal eyes. ${ }^{6}$

In cases where PACG coexists with cataract, the lens factor can be excluded by cataract extraction through phacoemulsification plus IOL implantation. This relieves both anterior segment crowding and secondary shallowing of the AC by increasing its depth and widening the angle, as well as reducing IOP. ${ }^{23,24}$

We observed significant increases in angle width and AC depth in the present study. These results corroborate those reported by Dada et al, who evaluated the effect of cataract extraction by phacoemulsification plus foldable IOL implantation on biometric determinants of the $\mathrm{AC}$ angle assessed with UBM. ${ }^{34}$ They detected a significant widening of AC angle after phacoemulsification. Moreover, Dada et al and Astushi et al performed a retrospective study and concluded that all parameters (AC depth, angle opening and trabecular ciliary process distance) were significantly increased as detected by UBM in PACG patients who underwent phacoemulsification. ${ }^{35,36}$

Hayashi et al also detected increases in ACD and angle width and reported improvement of aqueous outflow after cataract extraction and large lens removal from crowded ACs in patients with PACG. In addition, Bailiff et al stated that irrigation of cellular debris from the trabecular meshwork by phaco-irrigation aspiration may augment trabecular outflow. ${ }^{38}$

The present results showed a negative correlation between increased angle width and IOP elevation. These results coincide with those reported by Huang et al who found a correlation between IOP reduction and angle widening after phacoemulsification. Elevated IOP was detected in patients with narrow angles compared to those with wider angles who had lower IOPs. ${ }^{32}$

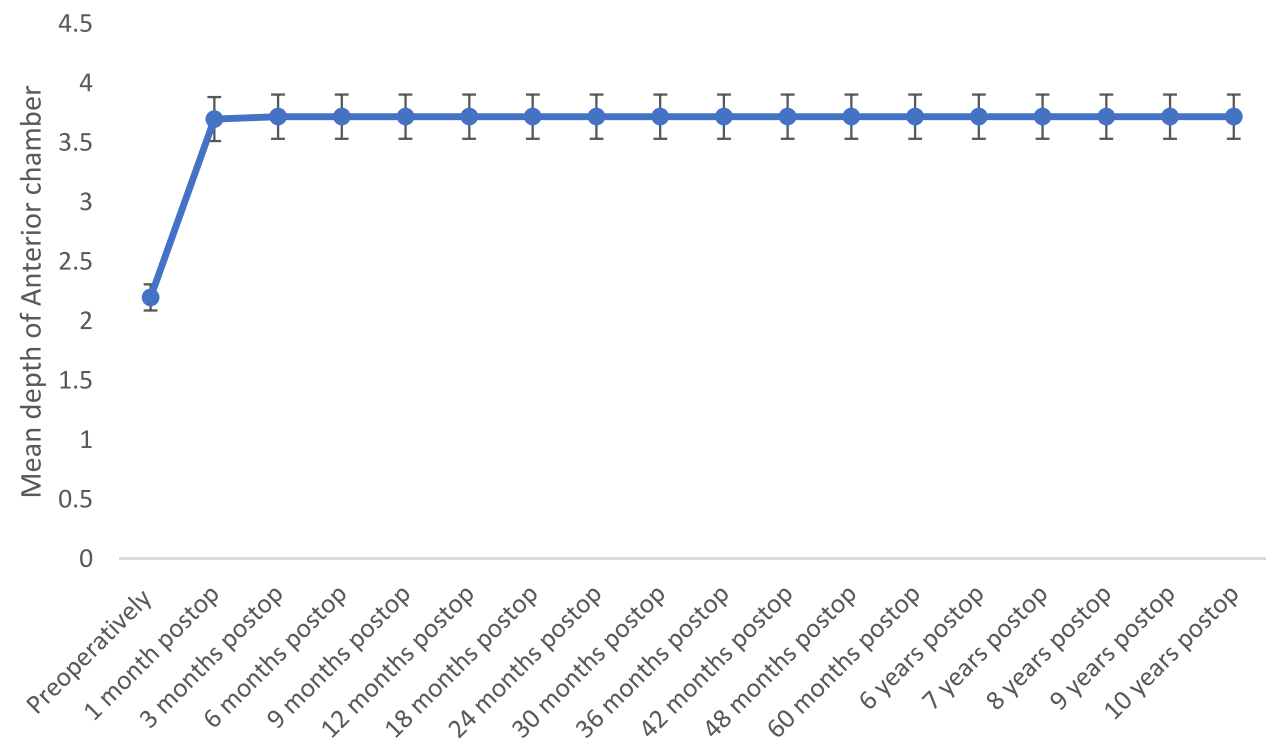

Figure 4 Line graph showing change in depth of $A C$ pre and postoperatively among the studied patients. 
Table 6 Change in Angle Width Pre and Postoperatively Among the Studied Patients

\begin{tabular}{|l|l|l|l|l|}
\hline \multirow{2}{*}{ Time } & \multicolumn{2}{|l|}{ Nasal Angle } & \multicolumn{2}{l|}{ Temporal Angle } \\
\cline { 2 - 5 } & Mean \pm SD & Range & Mean \pm SD & Range \\
\hline Preoperatively & $16.02 \pm 2.08$ & $11.7-20.1$ & $13.05 \pm 2.07$ & $8.8-17.2$ \\
I Month postop & $40.05 \pm 2.09$ & $35.9-44.2$ & $41.96 \pm 1.94$ & $37.8-45.23$ \\
\hline P & $\leq 0.00 I^{* *}$ & & $\leq 0.001 * *$ & \\
\hline
\end{tabular}

Note: $*_{p} \leq 0.001$ is statistically highly significant.

We also observed a negative correlation between preoperative AC depth and postoperative IOP. This is in accordance with the findings of Huang et al, who noted a correlation between a preoperative lens vault that makes the AC more shallow and IOP reduction after cataract surgery. ${ }^{39}$ Similarly, Liu et al identified a positive correlation between preoperative IOP value and AC depth and long-term IOP control. ${ }^{40}$ Furthermore, Nongpiur et al concluded that thicker lens and greater lens vault were obvious characteristics of PACG eyes. ${ }^{41}$

Our results revealed further IOP reduction in cases with higher preoperative IOP, more anti- glaucoma treatments, narrower iridotrabecular angle widths, and greater disc cupping. This is similar to the results that coincide with Shams et al, who found greater IOP reduction after phacoemulsification which in patients with higher preoperative IOP, more glaucoma medications, narrower iridotrabecular angle width, and greater extent of PAS formation and in eyes with evidence of glaucomatous optic neuropathy. ${ }^{27}$

In the current study, anti-glaucoma treatment use was significantly reduced, and $69.6 \%$ of cases were well controlled without medication postoperatively. Mierzejewski et al and Bhandari et al also reported decreased need for anti-glaucoma treatment after phacoemulsification cataract surgery. ${ }^{22,42}$
We specifically selected cases of PACG with PAS $\leq 180$ degrees because these patients are more suitable for phacoemulsification and do not require filtering surgery or combined phaco- trabeculectomy. This agrees with the recent Chinese guidelines for glaucoma diagnosis and management. Villavicencio and colleagues proposed that early phacoemulsification in this stage may be a good option to preserve the trabeculum, as it may become nonfunctional even after synechia lysis and angle widening in advanced stages of PAS. ${ }^{43}$ Euswas et al found further IOP reduction and reduced use of anti-glaucoma medications when phacoemulsification was performed in PACG patients with 180 compared to 270 degrees of PAS. ${ }^{44}$

Plateau iris constitutes the majority of non-pupillary block cases, whereas thick or anteriorly inserted irises are the minority of non-pupillary block causes of PACG. ${ }^{16}$ In plateau iris syndrome, anterior insertion of the iris root makes the iris more likely to be in contact with the trabeculum that affects the angle for synechia and closure. Peripheral iridotomy is less effective for this subset of patients. According to Mansoori et al, one-third of cases develop synechial angle closure after LPI due to the presence of plateau iris. ${ }^{8}$ In addition, Qiu et al concluded that $25 \%$ of PAC cases converted to PACG $>5$ years after LPI, and age and vertical CD ratio changes were identified as risk factors. ${ }^{45} \mathrm{He}$ et al found residual

Table 7 Correlation Between Preoperative AC Depth, Angle Width and IOP Among the Studied Patients

\begin{tabular}{|c|c|c|c|c|c|c|c|c|}
\hline \multirow[t]{2}{*}{ Variables } & \multicolumn{2}{|c|}{ AC Depth } & \multicolumn{2}{|c|}{ Angle Nasal } & \multicolumn{2}{|c|}{ Angle Temporal } & \multicolumn{2}{|l|}{ IOP } \\
\hline & $\mathbf{r}$ & $\mathbf{p}$ & $\mathbf{r}$ & $\mathbf{p}$ & $\mathbf{r}$ & $\mathbf{p}$ & $\mathbf{r}$ & $\mathbf{p}$ \\
\hline AC depth & & & 0.992 & $<0.001 * *$ & 0.985 & $<0.001 * *$ & -0.934 & $<0.001 * *$ \\
\hline Angle nasal & 0.992 & $<0.00 I^{* *}$ & & & 0.994 & $<0.001 * *$ & -0.925 & $<0.001 * *$ \\
\hline Angle temporal & 0.985 & $<0.001 * *$ & 0.994 & $<0.001 * *$ & & & -0.922 & $<0.00 I^{* *}$ \\
\hline IOP & -0.934 & $<0.001 * *$ & -0.925 & $<0.001 * *$ & -0.922 & $<0.001^{* *}$ & & \\
\hline Number of medications & -0.565 & $<0.001 * *$ & -0.653 & $<0.001 * *$ & -0.642 & $<0.001^{* *}$ & 0.655 & $<0.00 I^{* *}$ \\
\hline
\end{tabular}

Note: $*^{*} \mathrm{p} \leq 0.001$ is statistically highly significant.

Abbreviation: $r$, Pearson correlation coefficient. 


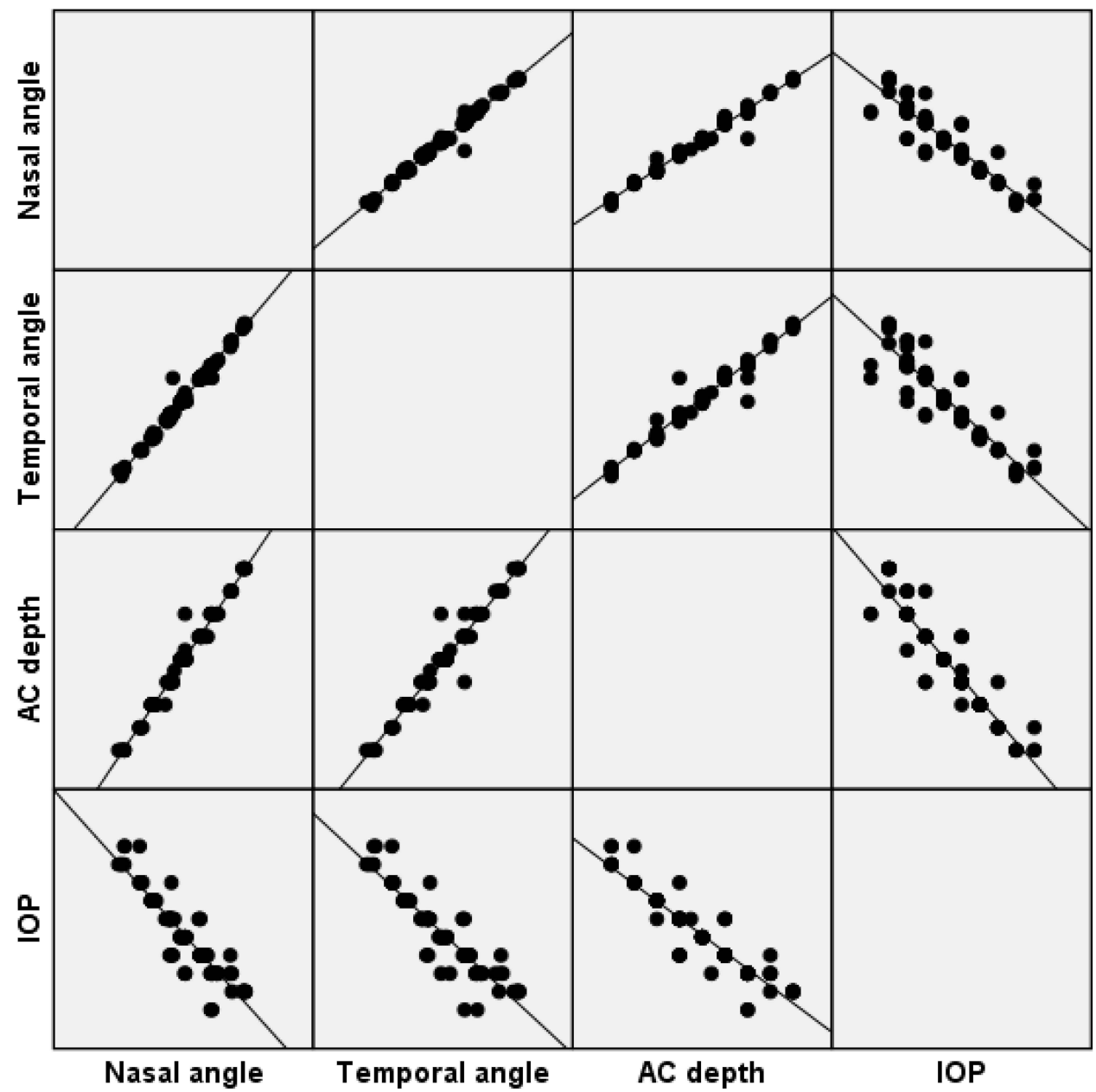

Figure 5 Scatter dot matrix showing correlations between IOP, AC depth, nasal and temporal angles among the studied patients.

iridotrabecular contact in $59 \%$ of cases after patent LPI. ${ }^{14}$ Phacoemulsification may provide a suitable option in these cases. ${ }^{8}$ Other studies concluded that phacoemulsification lowers IOP more than LPI in angle closure patients with visually significant cataract. ${ }^{26,34}$ Nonaka et al concluded that cataract surgery effectively resolved residual angle closure in $38.5 \%$ of cases after primary
LPI. ${ }^{15}$ Lam et al compared early phacoemulsification to peripheral iridectomy and found that the former approach was a more effective and definitive treatment for preventing IOP rise. ${ }^{26}$ Ang and Wells used optical coherence tomograph to assess outcomes and concluded that unsuccessful LPI was associated with a larger pre-laser angle closure. ${ }^{13}$ Furthermore, Napier and colleagues concluded

Table 8 Correlation Between I-Month Postoperative AC Depth, Angle Width and IOP Among the Studied Patients

\begin{tabular}{|l|l|l|l|l|l|l|l|l|}
\hline \multirow{2}{*}{ Variables } & \multicolumn{2}{l|}{ AC Depth } & \multicolumn{2}{l|}{ Angle Nasal } & \multicolumn{2}{l|}{ Angle Temporal } & \multicolumn{2}{l|}{ IOP } \\
\cline { 2 - 9 } & $\mathbf{r}$ & $\mathbf{p}$ & $\mathbf{r}$ & $\mathbf{p}$ & $\mathbf{r}$ & $\mathbf{p}$ & $\mathbf{r}$ & $\mathbf{P}$ \\
\hline AC depth & & & 0.159 & 0.11 & 0.148 & 0.137 & -0.178 & 0.073 \\
Angle nasal & 0.159 & 0.11 & & & 0.991 & $<0.00 I^{* *}$ & -0.974 & $<0.00 I^{* *}$ \\
Angle temporal & 0.148 & 0.137 & 0.991 & $<0.00 I^{* *}$ & & & -0.970 & $<0.00 I^{* *}$ \\
IOP & -0.178 & 0.073 & -0.974 & $<0.00 I^{* *}$ & -0.97 & $<0.00 I^{* *}$ & \\
Number of medications & -0.111 & 0.265 & -0.721 & $<0.00 I^{* *}$ & -0.749 & $<0.00 I^{* *}$ & 0.746 & $<0.00 I^{* *}$ \\
\hline
\end{tabular}

Note: $* *_{p} \leq 0.00 \mathrm{I}$ is statistically highly significant.

Abbreviation: r, Pearson correlation coefficient. 


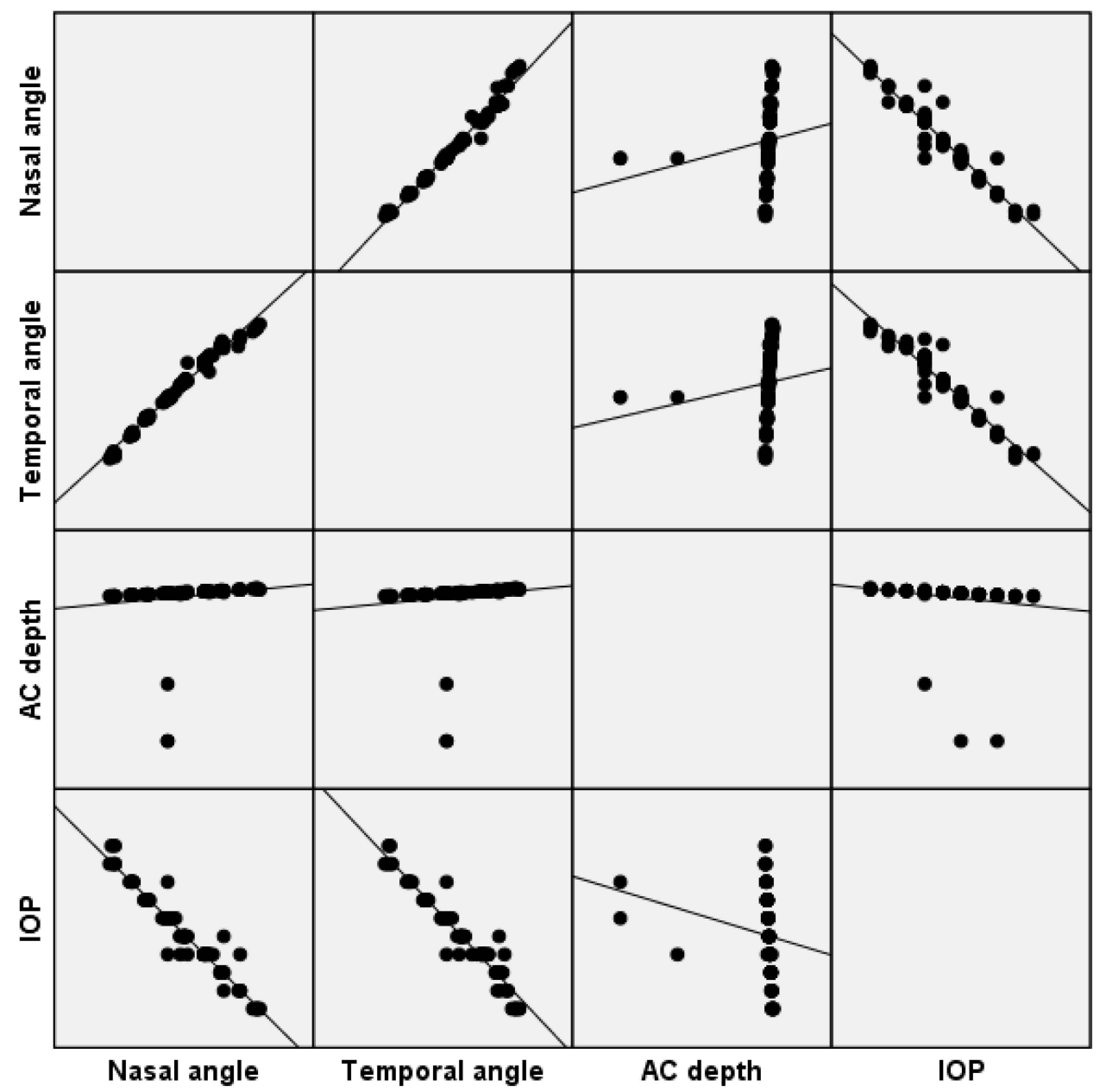

Figure 6 Scatter dot matrix showing correlations between IOP, AC depth, nasal and temporal angles one month postop among the studied patients.

that although LPI is a safe and effective mode of treatment, its effect is lost with time, and clear lens extraction achieves better clinical results and higher quality of life. They recommended primarily clear lens extraction in PAC and PACG eyes with IOP $>30 \mathrm{mmHg} .{ }^{5}$ In addition, Nongpiur detected a correlation between a higher proportion of plateau iris configuration and PACG severity. ${ }^{29}$

Table 9 Correlation Between Preoperative Lens Thickness and the Studied Parameters Among the Studied Patients

\begin{tabular}{|l|l|l|l|l|}
\hline \multirow{2}{*}{ Parameter } & \multicolumn{2}{|l|}{ Preoperatively } & \multicolumn{2}{l|}{ Postoperatively } \\
\cline { 2 - 5 } & $\mathbf{r}$ & $\mathbf{P}$ & $\mathbf{r}$ & $\mathbf{P}$ \\
\hline AC depth & -0.977 & $<0.00 I^{* *}$ & -0.105 & 0.293 \\
Nasal angle & -0.979 & $<0.00 I^{* *}$ & -0.976 & $<0.00 I^{* *}$ \\
Temporal angle & -0.973 & $<0.00 I^{* *}$ & -0.967 & $<0.00 I^{* *}$ \\
IOP & 0.939 & $<0.00 I^{* *}$ & 0.977 & $<0.00 I^{* *}$ \\
Medications & 0.671 & $<0.00 I^{* *}$ & 0.72 & $<0.00 I^{* *}$ \\
\hline
\end{tabular}

Note: $* * 0.001$ is statistically highly significant. Abbreviation: $r$, Pearson correlation coefficient.
The present study demonstrated long-term IOP stability following phacoemulsification for treatment of PACG. These results coincide with those reported by Husain and colleagues, who concluded that phacoemulsification has a lower rate of failure at 2 years compared to LPI (61.1\% success rate for LPI compared to $89.5 \%$ for phacoemulsification). ${ }^{34} \mathrm{Ng}$ et al concluded that $58 \%$ of

Table 10 Distribution of the Studied Patients According to Outcome of Surgery $(n=102)$

\begin{tabular}{|l|l|l|}
\hline \multicolumn{2}{|l|}{ N=102 } & $\%$ \\
\hline \multicolumn{2}{|l|}{ Success: } & 30.4 \\
\hline Qualified & 31 & 69.6 \\
Complete & 71 & 2.9 \\
\hline Need for another surgery: & 3 & \\
\hline Yes & 3 &
\end{tabular}




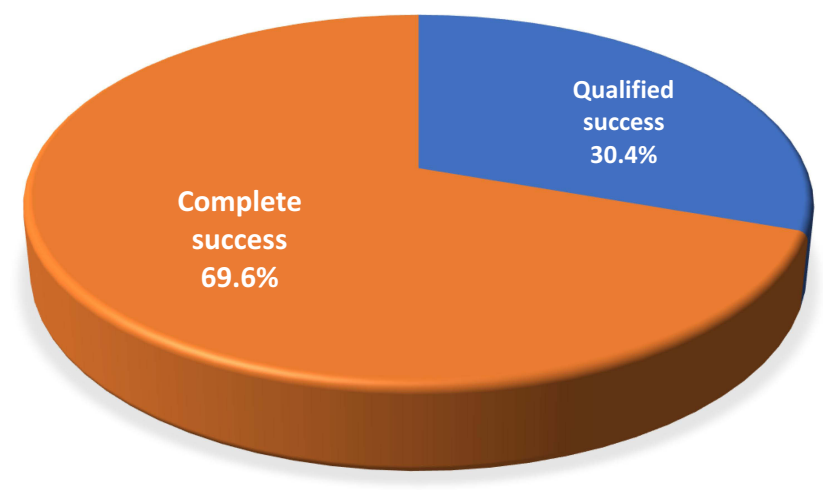

Figure 7 Pie chart showing distribution of the studied patients according to outcome.

angle closure glaucoma cases recur after LPI and that more studies are required to clarify the pathogenesis and develop a stepwise treatment approach. ${ }^{10}$

In accordance with our findings, Eslami et al concluded that phacoemulsification alone can be a good option to control IOP in cases of PACG with significant cataract. ${ }^{23}$ Conversely, Tham et al concluded that phacotrabeculectomy controls IOP better than phacoemulsification alone, but it may be associated with more complications. ${ }^{21}$ Goniosynechialysis is an adjunctive maneuver to phacoemulsification for treatment of PACG that added benefit according to Angmo et $\mathrm{al}^{46}$ but had no additive effect according to Lee and colleagues. ${ }^{47}$ In contrast to our results, Yudhasompop et al concluded that although phacoemulsification improved both IOP and visual acuity, the change in IOP was not significantly correlated with ACD or lens thickness. ${ }^{48}$

In our study, three $(2.9 \%)$ patients required surgical intervention to control IOP, and these cases were considered as failures. The remaining $97.1 \%$ of cases were well controlled with phacoemulsification alone or with one additional treatment. Few studies have investigated the

Table I I Postoperative Complications $(n=102)$

\begin{tabular}{|c|c|c|}
\hline & $N=102$ & $\%$ \\
\hline \multicolumn{3}{|l|}{ Complications } \\
\hline Hyphema & 5 & 4.9 \\
\hline Corneal edema & 40 & 39.2 \\
\hline Malignant glaucoma & 2 & 1.9 \\
\hline uveitis & 25 & 24.5 \\
\hline Corneal edema & 40 & 39.2 \\
\hline IOP spike & 12 & 11.8 \\
\hline Macular edema & 2 & 1.9 \\
\hline
\end{tabular}

long-term control of IOP in PACG patients after phacoemulsification. Hayashi et al found poor control among 3\% of cases over 2 years. $^{37}$ Shams and Forester reported worse IOP among 9\% of patients after 14 months. $^{27}$ Tham et al described worse IOP among $19.5 \%$ of patients after 2 years. $^{25}$ Lai et al found concluded that more medications may be required in up to $24 \%$ of patients after 18 months. ${ }^{49}$ Our results are most similar to those of Hayashi et al, and the high success rate may be explained by our selection of cases with $\leq 180$ degrees PAS. ${ }^{37}$

We observed several complications such as corneal edema, uveitis, malignant glaucoma, and hyphema, all of which are more common in PACG compared to open angle glaucoma. This may be related to anatomical characteristics of eyes with PACG, such as shallow AC, short axial length, endothelium effects of anti-glaucoma treatment, and a crowded AC that make surgical intervention more challenging. This has been reported by several groups. ${ }^{20,21,36,49}$ All complications resolved completely with conservative medical treatment and did not affect visual acuity in any patient.

To our knowledge, this is the first study to assess the long-term effect of early phacoemulsification to control IOP in PACG patients over 10 years. Limitations of this study include the absence of a control group and the fact that only early PACG cases were included.

\section{Conclusion}

Early phacoemulsification with IOL implantation can be considered as safe and effective for management of PACG cases with coexisting cataract. Its beneficial effects can persist up to 10 years.

\section{Acknowledgments}

The author thanks the Research Institute of ophthalmology (RIO) for support during the conduct of this study.

\section{Disclosure}

The author reports no conflicts of interest in this work.

\section{References}

1. Quigelly HA, Broman AT. The number of people with glaucoma worldwide in 2010 and 2020. Br J Ophthalmol. 2006;90:262-267. doi:10.1136/bjo.2005.081224

2. Wang F, Wu Z. Phacoemulsification versus combined phacotrabeculectomy in the treatment of chronic angle closure glaucoma with cataract: a meta-analysis. Int J Ophthalmol. 2016;9(4):597-603.

3. Shiota R, Dada T, Gupta R, Kakshminarayan P, Pandey R. Ultrasound biomicroscopy in the subtypes of primary angle closure glaucoma. $J$ Glaucoma. 2005;14:387-391. doi:10.1097/01.ijg.0000176934. 14229.32 
4. Mansori M, Ramezani F, Moghimi S, et al. Anterior segment optical coherence tomography parameters in phacomorphic angle closure and mature cataract risk factors in phacomorphic angle closure. IVOS. 2014;55:7403-7409.

5. Napier M, Azuara-Blanco A. Changing patterns in treatment of angle closure glaucoma. Curr Opin Ophthalmol. 2018;29(2):130-134. doi:10.1097/ICU.0000000000000453

6. George R, Paul P, Baskaran M, et al. Ocular biometry in occlude-able angles and angle closure glaucoma: a population based survey. $\mathrm{Br}$ J Ophthalmol. 2003;87:399-402. doi:10.1136/bjo.87.4.399

7. Taronogy P, Ho CL, Walton D. The role of the lens in the pathogenesis, prevention and treatment of angle closure glaucoma. Survey Ophthalmol. 2009;54(2):211-225. doi:10.1016/j.survophthal.2008.12.002

8. Mansoori T, Sarvepally V, Balakrishna N. Plateau iris in primary angle closure glaucoma: an ultrasound bio microscopy study. J Glaucoma. 2016;. 25(2):82-86. doi:10.1097/ IJG.0000000000000263

9. Stefen C, Iliescu D, Batras M, Timaru C, Simone A. Plateau iris diagnosis and treatment. Rom J Ophthalmol. 2015;59(1):14-18.

10. Ng WT, Morgan W. Mechanisms and treatment of primary angle closure: a review. Clin Exp Ophthalmol. 2012;40(4):218-228. doi:10.1111/j.1442-9071.2011.02604.x

11. Foster PJ, Buhrmann R, Quigely HA. The definition and classification of glaucoma in prevalence surveys. $\mathrm{Br} J$ Ophthalmol. 2002;86:238-242. doi:10.1136/bjo.86.2.238

12. Kim YY, Yoo C, Cho SY, Lee TE. Anterior segment parameters measured by ultrasound bio microscopy in the subtypes of angle closure. Acta Ophthalmol. 2016;94:s 256. doi:10.1111/j.17553768.2016.0509

13. Ang GS, Wells AP. Factors influencing laser peripheral iridotomy outcomes in white eyes. An anterior segment optical coherence tomography study. J Glaucoma. 2011;20(9):577-583. doi:10.1097/ IJG.0b013e3181f7b26f

14. He M, Friedman DS, Ge J, et al. Laser peripheral iridotomy in eyes with narrow drainage angles. Ultrasound bio-microscopy outcomes. The Liwan eye study. Ophthalmology. 2007;114(8):1513-1519. doi:10.1016/j.ophtha.2006.11.032

15. Nonaka A, Kondo T, Kikuchi M, et al. Angle widening and alteration of ciliary process configuration after cataract surgery for primary angle closure. Diaz Core Med J. 2006;113(3):437-441.

16. Yang $\mathrm{CH}$, Hung PT. Intraocular lens position and anterior chamber angle changes after cataract extraction in eyes with primary angle closure glaucoma. J Cataract Refract Surge. 1997;23:1109-1113. doi:10.1016/S0886-3350(97)80089-2

17. Belluci R, Perfetti S, Babighian S, Morselli S, Banomi L. Filtration and complications after trabeculectomy and after phacotrabeculectomy. Acta Ophthalmol Scand Suppl. 1997;224:44-45.

18. Aung T, Tow SL, Yap EU, Chan SP, Seah SK. Trabeculectomy in patients with chronic angle closure. Ophthalmol. 2000;107 (7):1298-1302. doi:10.1016/S0161-6420(00)00137-8

19. Quigelly HA, Friedman DS, Congdon NG. Possible mechanisms of primary angle closure and malignant glaucoma. J Glaucoma. 2003;12 (2):167-180. doi:10.1097/00061198-200304000-00013

20. Moghimi S, Hashemian H, Chen R, Johari M, Mohammadi M, Lin SC. Early phacoemulsification in patients with primary angle closure. J Curr Ophthalmol. 2015;27(3-4):70-75. doi:10.1016/j. joco.2015.12.001

21. Tham CY, Kwong YY, Baig N, Leung D, Li F, Lam D. Phacoemulsification versus trabeclectomy in medically uncontrolled chronic angle closure glaucoma without cataract. Ophthalmol. 2013;120(1):62-66. doi:10.1016/j. ophtha.2012.07.021

22. Bhandari S, Pandyal I, Khanal SP, Thapa SS. Effect of phacoemulsification surgery on various parameters in patients with glaucoma. Nepal J Ophthalmol. 2014;6(11):46-55. doi:10.3126/nepjoph. v6i1.10772
23. Eslami Y, Fakhraei G, Zaeri R, et al. Effect of cataract extraction on intraocular pressure in chronic angle closure glaucoma. J Curr Ophthmol. 2006;19(3):6-8.

24. Latifi G, Moghimi S, Eslami EY, Fakhraie G, Zaeri R, Lin S. Effect of phacoemulsification on drainage angle status in angle closure eyes with or without extensive peripheral anterior synechia. Eur J Ophthalmol. 2013;23(1):70-79. doi:10.5301/ejo.5000191

25. Tham CC, Kwong YY, Leung DY. Phacoemulsification versus combined phacotrabeculectomy in medically controlled chronic angle closure glaucoma with cataract. Ophthalmol. 2008;115 (12):2167-2173. doi:10.1016/j.ophtha.2008.06.016

26. Lam DS, Leung DY, Tham CY, et al. Randomized trial of early phacoemulsification versus peripheral iridotomy to prevent intraocular pressure rise after acute primary angle closure. Ophthalmology. 2008;115:1134-1140. doi:10.1016/j.ophtha.2007.10.033

27. Shams PN, Foster PJ. Clinical outcomes after lens extraction for visually significant cataract in eyes with primary angle closure. J Glaucoma. 2012;21:545-550. doi:10.1097/IJG.0b013e31821db1db

28. Armstrong JJ, Wasiuta E, Kiatos M, Mehta M, Huntik ML. The effects of phacoemulsification on intraocular pressure and topical medication use in patients with glaucoma. J Glaucoma. 2017;26 (6):511-522. doi:10.1097/IJG.0000000000000643

29. Nongpiur M, Verma S, Tun T, Wong T, Petrera S, Aung T. Plateau Iris and severity of primary angle closure glaucoma. Am J Ophthalmol. 2020;220:1-8. doi:10.1016/j.ajo.2020.07.033

30. Glaucoma group ophthalmology branch of Chinese medical association. Expert consensus on diagnosis and treatment of primary glaucoma in China. Chin J Ophthalmol. 2014;5:382-383.

31. Kaushik S, Kumar R, Jain R, Bansal R, Pandav S, Gupta A. Ultrasound biomicroscopic qualification of the change in anterior chamber angle following laser peripheral iridotomy in early chronic primary angle closure glaucoma. Eye. 2007;21:735-741. doi:10.1038/ sj.eye. 6702317

32. Huang G, Gonzalez E, Peng PH, et al. Anterior chamber depth, iridocorneal angle width and intraocular pressure changes after phacoemulsification: narrow versus open iridocorneal angles. Arch Ophthalmol. 2011;129(11):1283-1290. doi:10.1001/ archophthalmol.2011.272

33. Ishikawa H, Schuman J. Anterior segment imaging: ultrasound bio-microscopy. Ophthalmol Clin North Am. 2004;17(1):7-20. doi:10.1016/j.ohc.2003.12.001

34. Husain R, Gazzard G, Aung T, et al. Initial management of acute primary angle closure: a randomized trial comparing phacoemulsification with laser peripheral iridotomy. Ophthalmology. 2012;119:2274-2281. doi:10.1016/j.ophtha.2012.06.015

35. Dada T, Mohan S, Bali SJ, Bhartiya S, Sobti A, Pnda A. Ultrasound biomicroscopic assessment of angle parameters in patients with primary closure glaucoma undergoing phacoemulsification. Eur J Ophthalmol. 2011;5:559-565. doi:10.5301/EJO.2011.6287

36. Astushi N, Takehesia K, Kenji Y, Massahi F, Takuji I. Cataract surgery for residual angle closure after peripheral laser iridotomy. Ophthalmology. 2005;112(6):974-979. doi:10.1016/j. ophtha.2004.12.042

37. Hayashi K, Hayashi H, Nakao F, Hayashi F. Effect of cataract surgery on intraocular pressure control in glaucoma patients. J Cataract Refract Surg. 2001;27(11):1779-1786. doi:10.1016/S0886-3350(01) 01036-7

38. Bailifi S, Roure-Sobas C, Le -duff F, Kodjikian L. Aqueous humor contamination during phacoemulsification in a university teaching hospital. J Fr Ophthalmol. 2012;35(3):153-156. doi:10.1016/j. jfo.2011.05.007

39. Huang G, Gonzalez E, Lee R, Chen YC, He ML, Lin SC. Association of biometric factors with anterior chamber angle widening and intraocular pressure reduction after uneventful phacoemulsification for cataract. J Cataract Refract Surg. 2012;38:108-116. doi:10.1016/j. jcrs.2011.06.037 
40. Liu CJ, Cheng CY, Ko YC, Lau LI. Determinants of long term intraocular pressure after phacoemulsification in primary angle closure glaucoma. J Glaucoma. 2011;20(9):566-570. doi:10.1097/ IJG.0b013e3181efe1e9

41. Nongpiur ME, He M, Amerasinghe N, et al. Lens vault, thickness, and position in Chinese subjects with angle closure. Ophthalmology. 2011;118(3):474-479. doi:10.1016/j.ophtha.2010.07.025

42. Mierzejeweski A, Eliks I, Kaluzny B, Zygulska M, Harasimowicz B, Kalunzy K. Cataract phacoemulsification and intraocular pressure in glaucoma patients. Klin Oczna. 2008;110(1-3):11-17.

43. Villavicencio J, Arbelaez N, Lastra BR, et al. Primary outcomes of patients with chronic angle closure glaucoma treated with combined phacoemulsification, visco-goniosynechialysis

and endocyclo-photocoagulation. J Ophthalmol. 2019. Id 6378489. doi:10.1155/2019/6378489

44. Euswas A, Warrasak S. Intraocular pressure control following phacoemulsification in patients with chronic angle closure glaucoma. J Med Assoc Thai. 2005;88:S121-S125.

45. Qiu L, Yan Y, Wu L. Appositional angle closure and conversion of primary angle closure into glaucoma after laser peripheral iridotomy. Br J Ophthalmol. 2020;104(3):386-391. doi:10.1136/bjophthalmol2018-312956
46. Angmo D, Shakrawl J, Gupta B, Suresh Y, Pandey RM, Dada T. Comparative evaluation of phacoemulsification alone versus phacoemulsification with goniosynechialysis in primary angle closure glaucoma. A randomized controlled trial. Ophthalmol Glaucoma. 2019;2 (5):346-356. doi:10.1016/j.ogla.2019.05.004

47. Lee CK, Rho SS, Sung GJ, et al. Effect of goniosynechialysis during phacoemulsification on IOP in patients with medically well controlled chronic angle closure glaucoma. J Glaucoma. 2015;24 (6):405-409. doi:10.1097/IJG.0000000000000043

48. Yudhasompop N, Wangsupadilok B. Effects of phacoemulsification and intraocular lens implantation on intraocular pressure in primary angle closure glaucoma (PACG) patients. J Med Assoc Thai. 2012;95:557-560.

49. Lai JS, Tham CC, Chan JC. The clinical outcomes of cataract extraction by phacoemulsification in eyes with primary angle closure glaucoma and coexisting cataract, a prospective case series study. J Glaucoma. 2006;15(1):47. doi:10.1097/01.ijg.0000196619. $34368.0 \mathrm{a}$
Clinical Ophthalmology

\section{Publish your work in this journal}

Clinical Ophthalmology is an international, peer-reviewed journal covering all subspecialties within ophthalmology. Key topics include: Optometry; Visual science; Pharmacology and drug therapy in eye diseases; Basic Sciences; Primary and Secondary eye care; Patient Safety and Quality of Care Improvements. This journal is indexed on PubMed
Dovepress

Central and CAS, and is the official journal of The Society of Clinical Ophthalmology (SCO). The manuscript management system is completely online and includes a very quick and fair peer-review system, which is all easy to use. Visit http://www.dovepress.com/ testimonials.php to read real quotes from published authors. 